\title{
Terre, ethnicité et légitimité politique au Soudan
}

Catherine Miller, en collaboration avec François Ireton et Isabelle Dalmau (dir.), Land, Ethnicity and Political Legitimacy in Eastern Sudan, Le Caire, CÉDÉJ/DSRC, 2005, 502 p.

\section{Marc Lavergne}

\section{OpenEdition}

\section{Journals}

Édition électronique

URL : http://journals.openedition.org/transcontinentales/1453

DOI : $10.4000 /$ transcontinentales. 1453

ISBN : 978-2-8218-1408-0

ISSN : 1775-397X

Éditeur

Editions de la maison des sciences de l'homme

Édition imprimée

Date de publication : 30 juin 2006

Pagination : 174-176

ISBN : 2200-92169-1

ISSN : 1950-1684

Référence électronique

Marc Lavergne, «Terre, ethnicité et légitimité politique au Soudan », Transcontinentales [En ligne], 2 | 2006, document 12, mis en ligne le 15 décembre 2012, consulté le 23 septembre 2020. URL : http:// journals.openedition.org/transcontinentales/1453 ; DOI : https://doi.org/10.4000/transcontinentales 1453

Ce document a été généré automatiquement le 23 septembre 2020

Tous droits réservés 


\title{
Terre, ethnicité et légitimité politique au Soudan
}

\author{
Catherine Miller, en collaboration avec François Ireton et Isabelle \\ Dalmau (dir.), Land, Ethnicity and Political Legitimacy in Eastern Sudan, \\ Le Caire, CÉDÉJ/DSRC, 2005, 502 p.
}

\section{Marc Lavergne}

\section{RÉFÉRENCE}

Catherine Miller, en collaboration avec François Ireton et Isabelle Dalmau (dir.), Land, Ethnicity and Political Legitimacy in Eastern Sudan, Le Caire, CÉDÉJ/DSRC, 2005, 502 p.

1 Cette somme, consacrée à l'étude de deux régions contiguës de l'Est du Soudan Gedaref et Kassala -, est issue d'un programme collectif initié en 1988 qui a connu de nombreuses tribulations. Y sont traitées les relations entre l'accès à la terre, l'usage agro-pastoral de celle-ci et les rapports de production qui y président, d'une part et l'ethnicité, son fonctionnement et son instrumentation politique et sociale de l'autre. Le rôle du pouvoir colonial puis du pouvoir national central dans la construction ethnique et la manière dont ils ont forgé leur assise en instituant des pouvoirs locaux sont ensuite décryptés à travers les politiques de répartition des ressources foncières et de division tribale, ethnique et confrérique. Ces résultats constituent une moisson considérable, voire foisonnante. Elle apportera une contribution très utile non seulement à la connaissance de la région, mais surtout aux mécanismes fondamentaux de l'ethnogenèse et à la part qu'y ont pris les pouvoirs d'État dans l'histoire du Soudan depuis le XIX ${ }^{e}$ siècle.

2 Treize contributions de chercheurs soudanais et européens ont été réunies et mises en forme par Catherine Miller, socio-linguiste, épaulée par François Ireton, démographe, et Isabelle Dalmau, anthropologue.

3 L'introduction de C. Miller présente le cadre géographique et administratif de la région, puis développe le contexte politique sous l'angle, qui sert de fil conducteur au livre, du 
"Native Administration System », qualifié de "Phénix de la vie politique soudanaise ». Cet exposé met en lumière la politique du Front national islamique au pouvoir à l'égard des élites et des institutions locales, qui a pour but de réduire toute structure autonome (confréries religieuses, communautés de commerçants) capable de s'opposer au pouvoir central et aux groupes d'intérêts qu'il privilégie. D'où une stratégie de cooptation des éléments et une lutte radicale contre ceux qui s'obstinent à s'opposer au pouvoir ou dont les intérêts entrent en conflit avec lui. Un développement montre comment l'agriculture mécanisée a été privilégiée depuis les années 1940 par l'État, mettant ainsi en péril la survie des groupes pastoraux nomades, marginalisés ou contraints de développer des stratégies alternatives. L'introduction s'achève sur une revue des ethnies, de l'ethnogenèse et des relations interethniques dans la région, présentant les études de cas qui suivent.

4 En ouverture de la deuxième partie, consacrée aux «dynamiques politiques et économiques ", F. Ireton dresse un tableau démographique, fondé sur l'examen très technique des recensements de la population. Trois contributions de jeunes chercheurs soudanais se signalent ensuite par leur finesse d'analyse, leur prise en compte de la dimension historique, et leur pertinence pour la compréhension des logiques et des stratégies de pouvoir, du niveau de l'État à celui des structures locales. Inas Ahmed, politologue, montre comment le régime islamiste s'efforce de réduire l'influence des chefferies tout en récupérant à son profit les chefferies tribales ; son article est éclairé par celui que son collègue Amani M. El-Obeid consacre à l'étude des confréries soufies. Munzoul A. M. Assal, anthropologue, étudie les relations entre politique et économie à Gedaref, dévoilant une économie spéculative, accapareuse de terres fertiles et dévastatrice de l'environnement, favorisée par les gouvernements successifs qui y voient une ressource pour les groupes dirigeants et leurs relais locaux. Le dénuement de la région, en termes d'infrastructures et d'alphabétisation par exemple, n'en est que plus frappant. Ce qui est décrit là s'identifie à un système colonial, caractéristique de l'économie de prédation dont se contentent les pouvoirs soudanais depuis l'époque des sultanats du Darfunj et du Darfour. Les deux piliers en sont l'agriculture extensive destinée à l'exportation et désormais, en d'autres régions du pays, l'extraction pétrolière.

5 L'article de l'anthropologue Christian Delmet intitulé «Le système d'administration tribale au Soudan oriental: de la liquidation à la renaissance " offre une histoire générale de l'administration tribale au Soudan puis une analyse fondée sur des enquêtes récentes plus localisées. On peut regretter que l'occasion n'ait pas été saisie d'établir une comparaison avec d'autres parties du pays, où l'attribution ou non de territoires propres, les «dar ", à des groupes tribaux peut être, comme au Darfour, à l'origine des conflits dévastateurs d'aujourd'hui.

6 La troisième partie, intitulée "l'histoire de la population et la construction ethnique ", consiste en une déclinaison d'études de cas portant sur des groupes tribaux ou ethniques de la région, dont la genèse est retracée avec un luxe de détails tirés le plus souvent des archives du Sudan Office britannique.

7 À travers la " success story » des Arabes Rachaïda venus d'Arabie au xIX siècle, et qui ont réussi, en se reconvertissant dans la spécialisation chamelière et le commerce transfrontalier, à se faire une place au détriment des Béja hadendowa autochtones, Iman Bushra, politologue, rappelle le fonctionnement interne des tribus arabes et montre comment l'État soudanais s'appuie sur le local, sans que celui-ci ne parvienne 
en retour à l'influencer. L'État combine avec habileté clientélisme et népotisme avec la rente pétrolière pour s'assurer un contrôle serré de l'opinion tribale et des relations intertribales. Les éleveurs lahawiyin implantés sur les rives de l'Atbara illustrent le cas de tribus qui réclament un "dar " et un "nazirat ", c'est-à-dire un territoire et un pouvoir en propre pour s'affirmer, tandis que les Bawadra, forts de leur succès dans l'agriculture mécanisée, n'éprouvent pas le besoin de cette reconnaissance formelle.

La situation des Arabes nomades Ahamda, dispersés en fractions minoritaires depuis leur départ des rives du Nil Blanc, est relatée par Barbara Casciarri, anthropologue, qui, après un rappel utile sur le concept de tribu, montre le rôle des Britanniques dans la redéfinition des tribus et la division des Ahamda en deux groupes aux destins différents, l'un dans la province de Khartoum, l'autre sur le périmètre de La Nouvelle Halfa.

Deux articles consacrés aux «Fellata», ou à proprement parler aux descendants d'immigrants d'Afrique de l'Ouest (Nigeria) installés depuis plusieurs générations, voire depuis des siècles, dans la région du Nil Bleu et plus récemment de Gedaref, rectifient nombre d'idées reçues sur ces communautés mal connues et toujours considérées comme allogènes, bien qu'elles jouent un rôle économique et social croissant.

Enfin, deux études sont consacrées aux changements dans les relations inter-genres dans une communauté pastorale et au rôle de la poésie dans l'éthique de la société béja, par ailleurs largement absente, tout comme les Nubiens réinstallés à La Nouvelle Halfa, ou d'autres communautés immigrées récemment (Nouba, Sud-Soudanais, Darfouriens...), de cette sélection d'études de cas.

11 Si l'ouvrage souffre d'une construction a posteriori qui ne parvient pas toujours à éviter les écueils de la compilation, le titre affiché n'en est pas moins traité de manière très éclairante. On peut toutefois regretter que l'approche retenue ait conduit à écarter des thèmes comme l'urbanisation et la gestion urbaine, le développement de l'industrie, du commerce et des transports, l'inscription de la région dans l'ensemble soudanais, ses relations avec le pouvoir central et avec le voisinage abyssin, qui auraient donné accès à une représentation plus complète de la réalité humaine de la région. D'un point de vue plus formel enfin, on peut déplorer quelques maladresses de présentation et l'absence d'un réseau de diffusion. Le souci louable qu'a le CÉDÉJ de procéder lui-même à une publication en anglais, de manière à rendre ce travail accessible à un large public, trouve ici ses limites.

\section{AUTEURS}

\section{MARC LAVERGNE}

CNRS Groupe de recherche et d'études,sur la Méditerranée et le Moyen-Orient (GREMMO) - Université de Lyon 2 